\title{
Phase-Binarized Spin Hall Nano-Oscillator Arrays: Towards Spin Hall Ising Machines
}

\author{
Afshin Houshang, ${ }^{1, \dagger}$ Mohammad Zahedinejad, ${ }^{1, \dagger}$ Shreyas Muralidhar $\odot,{ }^{1}$ Jakub Chęciński® ${ }^{1,2}$ \\ Roman Khymyn, ${ }^{1}$ Mona Rajabali, ${ }^{1}$ Himanshu Fulara $\odot,{ }^{1}$ Ahmad A. Awad®,${ }^{1}$ Mykola Dvornik®, ${ }^{3}$ and \\ Johan Åkerman $\oplus^{1, *}$ \\ ${ }^{1}$ Physics Department, University of Gothenburg, Gothenburg 412 96, Sweden \\ ${ }^{2}$ AGH University of Science and Technology, Institute of Electronics, Al. Mickiewicza 30, Kraków 30-059, Poland \\ ${ }^{3} \mathrm{NanOsc}$ AB, Electrum 229, Kista 164 40, Sweden
}

(Received 17 August 2021; revised 29 October 2021; accepted 9 December 2021; published 3 January 2022; corrected 25 January 2022)

\begin{abstract}
Ising machines (IMs) are physical systems designed to find solutions to combinatorial optimization (CO) problems mapped onto the IM via the coupling strengths between its binary spins. Using its intrinsic dynamics and different annealing schemes, the IM relaxes over time to its lowest-energy state, which is the solution to the $\mathrm{CO}$ problem. IMs have been implemented on different platforms, and interacting nonlinear oscillators are particularly promising candidates. Here we demonstrate a pathway towards an oscillatorbased IM using arrays of nanoconstriction spin Hall nano-oscillators (SHNOs). We show how SHNOs can be readily phase binarized and how their resulting microwave power corresponds to well-defined global phase states. To distinguish between degenerate states, we use phase-resolved Brillouin-lightscattering microscopy and directly observe the individual phase of each nanoconstriction. Micromagnetic simulations corroborate our experiments and confirm that our proposed IM platform can solve CO problems, showcased by how the phase states of a $2 \times 2$ SHNO array are solutions to a modified max-cut problem. Compared with the commercially available D-Wave Advantage ${ }^{\mathrm{TM}}$, our architecture holds significant promise for faster sampling, substantially reduced power consumption, and a dramatically smaller footprint.
\end{abstract}

DOI: 10.1103/PhysRevApplied.17.014003

\section{INTRODUCTION}

Conventional computers based on a Von Neumann architecture are unable to efficiently address a certain class of problems known as combinatorial optimization (CO) problems [1]. These are by no means rare, and manifest themselves in some critically important areas such as business operations, manufacturing, research, integratedcircuit design, protein folding, DNA sequencing, discovery of new medicines, and efficient big-data clustering, to name a few. In parallel, Moore's law is continuing to slow down and approach its limits, making it even more vital to rethink current computation schemes and explore alternative paradigms. One important avenue in that regard

\footnotetext{
*johan.akerman@physics.gu.se

${ }^{\dagger}$ These authors contributed equally to this work.
}

Published by the American Physical Society under the terms of the Creative Commons Attribution 4.0 International license. Further distribution of this work must maintain attribution to the author(s) and the published article's title, journal citation, and DOI. Funded by Bibsam. is the concept of natural computing (NC), where a specific problem is mapped onto the physics of a system. The system then evolves, either naturally or under control, towards its ground state, which is the solution of the given problem. Within the realm of NC, many proposals have been put forward, among which quantum computers, Ising machines, and even combinations of both are currently the most studied examples.

The Ising model is a mathematical model of magnetic order in a discrete system of two-state variables, also known as spins, $s_{i} \in\{-1,+1\}$. In essence, the model postulates that the spins in a lattice are arranged in such a way as to minimize the following Hamiltonian:

$$
H(\vec{s})=-\sum_{i=1}^{N} h_{i} s_{i}-\sum_{i=1}^{N} \sum_{j \neq i}^{N} J_{i j} s_{i} s_{j},
$$

where $\vec{s}$ is the vector of spin variables and $N$ is its length. There are two types of contributions to the Ising Hamiltonian: (1) an interaction with the external field, represented by so-called bias terms, $h_{i}$, and (2) interactions between spins $i$ and $j$, described by the values of $J_{i j}$. What makes 
this model particularly technologically relevant is that the minimization of the Ising Hamiltonian is equivalent to a quadratic unconstrained binary optimization (QUBO) problem [2], which operates on binary variables $x_{i} \in\{0,1\}$. Consequently, a large set of nondeterministic-polynomialtime-hard combinatorial optimization problems, e.g., the traveling salesman problem, the graph-coloring problem, the max-cut problem, and integer factorization, can be directly mapped onto the Ising model via appropriate choices of $h_{i}$ and $J_{i j}$ [3-5]. It is therefore particularly interesting if a physical system is designed with the sole purpose of minimizing Eq. (1).

An IM is any hardware device whose node interactions can be described by an Ising Hamiltonian and is tasked with finding its ground state, which represents the solution to a specific $\mathrm{CO}$ problem defined by the connection strengths between the nodes and their biases. Guided by the individual interactions between all nodes, which are evaluated continuously through the inherent parallelism of the system, the IM wanders through its multivariate energy landscape. With the help of different types of annealing schemes to avoid local minima, the IM finds its global minimum in a time up to factorially faster compared with serial computation. Given the importance of CO problems and the efficiency of IMs in solving them, there have been multiple recent hardware implementations, such as ones using quantum annealers [6,7], CMOS annealers [8-10], nanomagnet network arrays [11], electronic oscillators [12,13], and laser networks $[14,15]$.

Launched in 2011, D-Wave One (Rainier) was a quantum annealing IM using 128 superconducting qubits, later superseded by D-Wave 2000Q, with 2048 qubits, and the D-Wave Advantage System (Pegasus), with 5640 qubits and the ability to solve problems with up to one million variables using a combination of classical and quantum solvers. Like most quantum hardware, it requires millikelvin temperatures and large cryogenic facilities with tens of kilowatts of power consumption, comes with a high nonstandard production cost, and does not scale well. Coherent Ising machines (CIMs), implemented using externally pumped optical parametric oscillators [16], are room-temperature alternatives, which avoid the cryogenic requirements and are claimed to outperform D-Wave 2000Q in solving large-scale max-cut problems [17]. However, they too face serious challenges in terms of scaling, footprint, and power consumption. In addition, CIMs require optical tables and kilometer-long optical fibers to accommodate all of the time-multiplexed optical parametric oscillators required. Once these systems have been built and the operating frequency has been set, no further tuning is available unless new hardware with a new architecture replaces the existing one.

To provide a room-temperature digital CMOS-based alternative, Fujitsu, in 2018 [18], announced a digital annealer unit (DAU) using a field-programmable gate array (FPGA), offering 8192 simulated qubits with all-to-all connectivity, allowing all qubits to exchange signals freely. Thanks to their superior connectivity, DAUs are capable of dealing with sizeable real-world-scale CO problems. However, they still rely on the Von Neumann computing paradigm, together with the added reconfigurability of FPGAs, and not on the nature of a physical system. It is hence not likely that it will be possible to scale this approach to much larger systems [11].

Inspired by both the optical pumping mechanism and the practical nature of ordinary CMOS electronics, Wang and Roychowdhury [19] proposed nonlinear-oscillatorbased IMs based on off-the-shelf electronics. In particular, the phase dynamics of a network of coupled oscillators can minimize a scalar, so-called Lyapunov, function [20], which serves as a measure of the stability of the network. If the network is locked to an external signal with a comparable frequency, the phases of each individual oscillator lock deterministically to that of the external perturbation [Fig. 1(c)], and their phase dynamics can be accurately described by the Gen-Adler model $[21,22]$. However, when subjected to subharmonic injection locking (SHIL) at twice their frequency, the individual phases of the oscillators in the network become bistable and can take on only binarized values of either 0 or $\pi$ [23], as depicted in Fig. 1(d). In this way, SHIL provides a discretization of the continuous phases that conforms with the binary Ising model Eq. (1). Under SHIL, the Lyapunov function directly translates into the QUBO equivalent of Eq. (1) as follows (see the Appendix for details):

$$
\begin{aligned}
E(\vec{x})= & -\sum_{i=1}^{N} \sum_{j \neq i}^{N} 4 \tilde{J}_{i j} x_{i} x_{j} \\
& +\sum_{i=1}^{N}\left[\sum_{j \neq i}^{N} 2 \tilde{J}_{i j}-\frac{\pi\left(2 f_{i}-f_{\text {inj }}\right)}{f_{i}}\right] x_{i},
\end{aligned}
$$

where $f_{\text {inj }}$ and $f_{i}$ are the frequency of the rf injection and the free-running frequencies of the oscillators, $\tilde{J}_{i j}$ is the dimensionless coupling between oscillators $i$ and $j$, and $x_{i}=\phi_{i} / \pi$, where $\phi_{i}$ is the phase of oscillator $i$ with respect to a defined reference. Equation (2) suggests that the linear QUBO terms can be independently changed by controlling the free-running frequencies of the oscillators, while tuning the oscillator-to-oscillator interaction changes both the quadratic and the linear terms. So, combining both controls enables programming of a particular QUBO model. As the system of coupled and biased SHIL oscillators evolves towards a stable state minimizing Eq. (2), possibly according to some annealing schedule, it essentially operates as an IM.

Wang and Roychowdhury then demonstrated a printedcircuit-board prototype using a network of 240 resistively coupled CMOS $L C$ oscillators with a maximum of 1200 

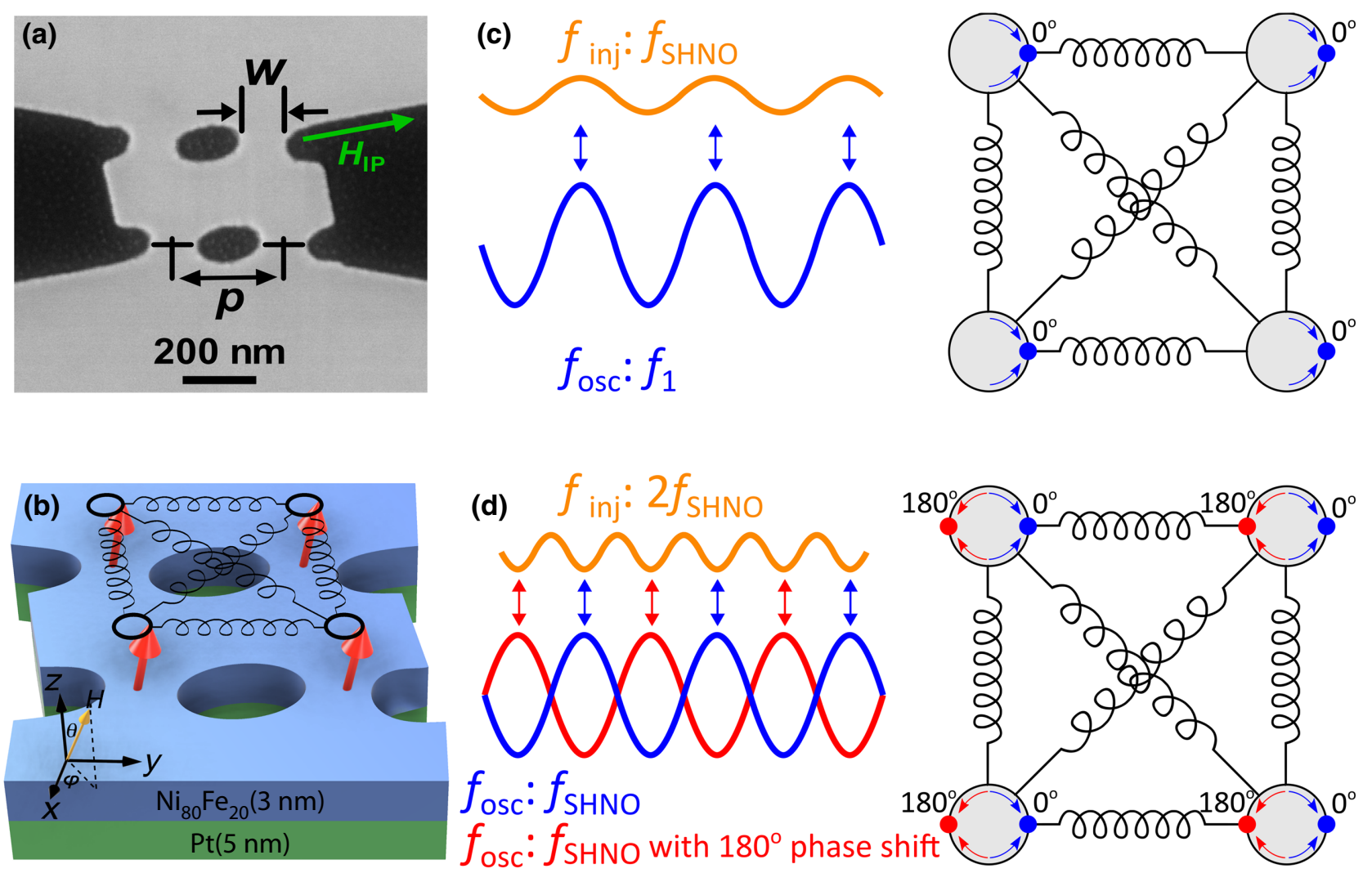

FIG. 1. Injection locking and principle of phase-binarized spin Hall nano-oscillator (SHNO) arrays. (a) Scanning-electronmicroscope image of a $2 \times 2$ SHNO array fabricated from a $\mathrm{Pt}(5 \mathrm{~nm}) / \mathrm{Ni}_{80} \mathrm{Fe}_{20}(3 \mathrm{~nm})$ bilayer (Hf dusting layer not shown). The width of each SHNO is $w=120 \mathrm{~nm}$, and their separation (pitch) is $p=300 \mathrm{~nm}$. $H_{\mathrm{IP}}$ shows the in-plane direction of the external magnetic field, $H$. (b) Schematic illustration of the SHNO array in (a), with red arrows showing the local magnetization vector and springs representing their couplings. (c) Ordinary first-harmonic injection locking $\left(f_{\text {inj }} \sim f_{\text {SHNO }}\right.$ ), where all SHNOs are locked in phase to the external source. (d) Second-harmonic injection locking (SHIL at $f_{\text {inj }} \sim 2 f_{\text {SHNO }}$ ) allows the oscillators to acquire one of two possible phase states separated by $180^{\circ}$, giving rise to phase binarization.

connections, achieving a solution time of $1 \mathrm{~ms}$ for a moderate operating frequency of $1 \mathrm{MHz}$ and a very modest power consumption of only $5 \mathrm{~W}$ [24]. In a similar demonstration, a network of four all-to-all-coupled $L C$ oscillators was shown to solve max-cut problems of small size [13]. Both reports forecast a significant boost in processing speed and solution quality if the coupled oscillators can be realized in large numbers and operate at gigahertz frequencies.

Spin Hall nano-oscillators (SHNOs) have recently emerged as one of the most promising CMOS compatible nano-oscillator technologies that can operate at several tens of gigahertz [25-31]. The most straightforward design of them requires only a nanoconstriction in a ferromagnet (FM)/heavy-metal bilayer [25], or even in a single FM layer [32]. SHNOs combine the following unique set of features that makes them one of the most promising routes towards truly miniaturized, ultrafast, and large-scale oscillator-based IMs: (a) their frequencies are highly tunable by means of an applied magnetic field, voltage-controlled magnetic anisotropy, and a bias current [33-36], enabling efficient control over the linear QUBO terms; (b) SHNOs can be coupled directly via spin waves [37] and/or magnetodipolar interactions [38], or indirectly, e.g., by electrical means, providing various ways to tune the quadratic QUBO terms; (c) they are readily injection locked to external microwave currents [36,39,40]; (d) they are intrinsically noisy [41], which should make the corresponding IMs less susceptible to the local-minima problem [42]; (e) they operate at up to $26 \mathrm{GHz}[30,33]$; (f) they have a truly nanoscale footprint, with lateral dimensions down to only $20 \mathrm{~nm}$ [43]; and (g) they have been recently shown to scale up to 64 oscillators arranged in square two-dimensional arrays [44].

Here we report on a significant milestone towards developing a miniaturized Ising machine, based on SHNOs. Firstly, we demonstrate robust phase binarization of $1 \times 2$ and $2 \times 2$ SHNO arrays using secondharmonic microwave current-injection locking. The phase binarization manifests itself as distinct microwave output power levels, which are readily distinguished using electrical means. We observe switching between different states induced by the injected power level and by 
sweeping the frequency of the injected signal. We then use phase-resolved Brillouin-light-scattering (phase-BLS) microscopy to directly observe the individual phases of the precessing magnetization in each nanoconstriction. Finally, we demonstrate that our devices can be considered as magnetic-field-tunable, fixed-function IMs tasked with solving a variant of the max-cut combinatorial optimization problem.

\section{EXPERIMENTAL METHOD}

\section{A. Fabrication}

The 2D SHNO arrays used in the present work are based on a trilayer of $\mathrm{Ni}_{80} \mathrm{Fe}_{20}(3 \mathrm{~nm}) / \mathrm{Hf}(0.5 \mathrm{~nm}) / \mathrm{Pt}(5 \mathrm{~nm})$ deposited on a high-resistivity silicon substrate (greater than $10 \mathrm{k} \Omega \mathrm{cm}$ ) using an ultrahigh-vacuum sputtering machine. The SHNO patterns are defined in a hydrogen silsesquioxane (HSQ) electron-beam resist and later transferred to the metallic trilayer using an ion-beam etching machine. The electrical top contacts, including a coplanar waveguide, are defined by optical lithography, followed by removal of the HSQ, deposition of a $1-\mu \mathrm{m}$-thick layer of $\mathrm{Cu}(980 \mathrm{~nm}) / \operatorname{Pt}(20 \mathrm{~nm})$, and liftoff in a resist remover. The fabrication of 2D SHNO arrays is detailed by Zahedinejad et al. [44].

\section{B. Microwave measurements and SHIL}

Microwave measurements are carried out at room temperature using a custom-built probe station, where the sample is mounted at fixed in-plane and out-of-plane angles of $\phi=24^{\circ}$ and $\theta=78^{\circ}$, respectively. A direct positive electric current, $I_{\mathrm{dc}}$, is then injected through the dc port of a high-frequency bias- $\mathrm{T}$ to induce auto-oscillations. Through a microwave circulator connected to the highfrequency port of the spectrum analyzer used, a rf current from a Rhode \& Schwarz $(10 \mathrm{~Hz}-20 \mathrm{GHz})$ signal source is injected into the auto-oscillating sample. The resulting signal is then amplified by a low-noise amplifier with a gain greater than or equal to $56 \mathrm{~dB}(4-10 \mathrm{GHz})$ and subsequently recorded using a spectrum analyzer from Rhode \& Schwarz $(10 \mathrm{~Hz}-40 \mathrm{GHz})$ with a low-resolution bandwidth of $300 \mathrm{kHz}$. A schematic illustration of the measurement setup was presented by Zahedinejad et al. [36].

\section{Phase-resolved BLS microscopy}

Phase-resolved BLS microscopy is performed by modulating the phase of the incoming light using an electro-optic modulator (EOM) at half the frequency $(f)$ of the injection signal $(2 f)$ applied to the SHNO array. The inelastically scattered outgoing light, carrying phase information from the oscillator, interferes with the elastically scattered light beam, whose phase $(\phi)$ can be controlled by a phase shifter (with a resolution of $5.6^{\circ}$ ) attached to the EOM. The resulting BLS signal is then the difference in phase between the light (known) and the oscillator (unknown). The signal is collected and analyzed in a six-pass tandem FabryPerot interferometer and detected using a single-channel avalanche photodiode. High spatial resolution is obtained at the expense of wave-vector resolution by using a highnumerical-aperture microscope objective, which forms a tight focus of the probe beam down to the diffraction limit (with a beam diameter of approximately $300 \mathrm{~nm}$ ). A three-axis nanometer-resolution stage, along with active stabilization using software provided by THATec Innovation $\mathrm{GmbH}$, makes it possible to perform very precise linear scans.

\section{RESULTS}

A SEM image of a $2 \times 2$ array is shown in Fig. 1(a). All devices are made from a bilayer of $\operatorname{Pt}(5 \mathrm{~nm}) / \mathrm{Ni}_{80} \mathrm{Fe}_{20}(3$ $\mathrm{nm}$ ), in which dusting the interface with an ultrathin $\mathrm{Hf}$ layer of thickness $0.5 \mathrm{~nm}$ reduces the damping in the $\mathrm{Ni}_{80} \mathrm{Fe}_{20}$ [35]. The width of all nanoconstrictions is $w=$ $120 \mathrm{~nm}$, and the pitch (separation) is $p=200 \mathrm{~nm}$ for the $1 \times 2$ array and $p=300 \mathrm{~nm}$ for the $2 \times 2$ array (see the detailed fabrication method in Ref. [44]). A magnetic field $H$ is applied, with an out-of-plane angle $\theta$ and an in-plane angle $\phi$; throughout this study, $\theta=78^{\circ}$ and $\phi=24^{\circ}$. In the SEM image, the in-plane component $H_{\mathrm{IP}}$ is explicitly shown. Figure 1(b) shows a schematic illustration of a $2 \times 2$ SHNO array, in which the red arrows show each individual oscillator and the springs illustrate the couplings between individual oscillators.

Figure 2(a) shows the power spectral density (PSD) of a $1 \times 2$ array, running at $I_{\mathrm{SHNO}}=3 \mathrm{~mA}$ in a field of $H=$ $6400 \mathrm{Oe}$, as the injected power, $P_{\mathrm{IL}}$, is ramped from -30 to $0 \mathrm{dBm}$. The SHNOs start out in a mutually synchronized state, which in this particular regime is possible due to the intrinsic long-range magnetodipolar coupling between oscillators [38]. When $P_{\mathrm{IL}}$ reaches a threshold of about $-16 \mathrm{dBm}$, the SHNOs lock intermittently to the external source. This injection locking gradually grows stronger and is quite stable above $-13 \mathrm{dBm}$, as evidenced by a substantially lower linewidth and a much higher peak power. When the injected power is increased further to $-10 \mathrm{dBm}$, the peak power splits into a high- and a low-power branch without any significant change in the linewidth. The two branches increasingly diverge with increasing $P_{\mathrm{IL}}$, and the peak power switches a few times between its two states on a slow timescale while remaining stable within each branch in between jumps. The timescale for the switching appears to slow down with increasing $P_{\mathrm{IL}}$, and above $-5 \mathrm{dBm}$ the SHNO remains in the lower branch for the remainder of the measurement.

The observed behavior is consistent with phase binarization in oscillator networks under SHIL, and we can essentially perform annealing by ramping up the injected power until the system reaches its preferred phase state, in 

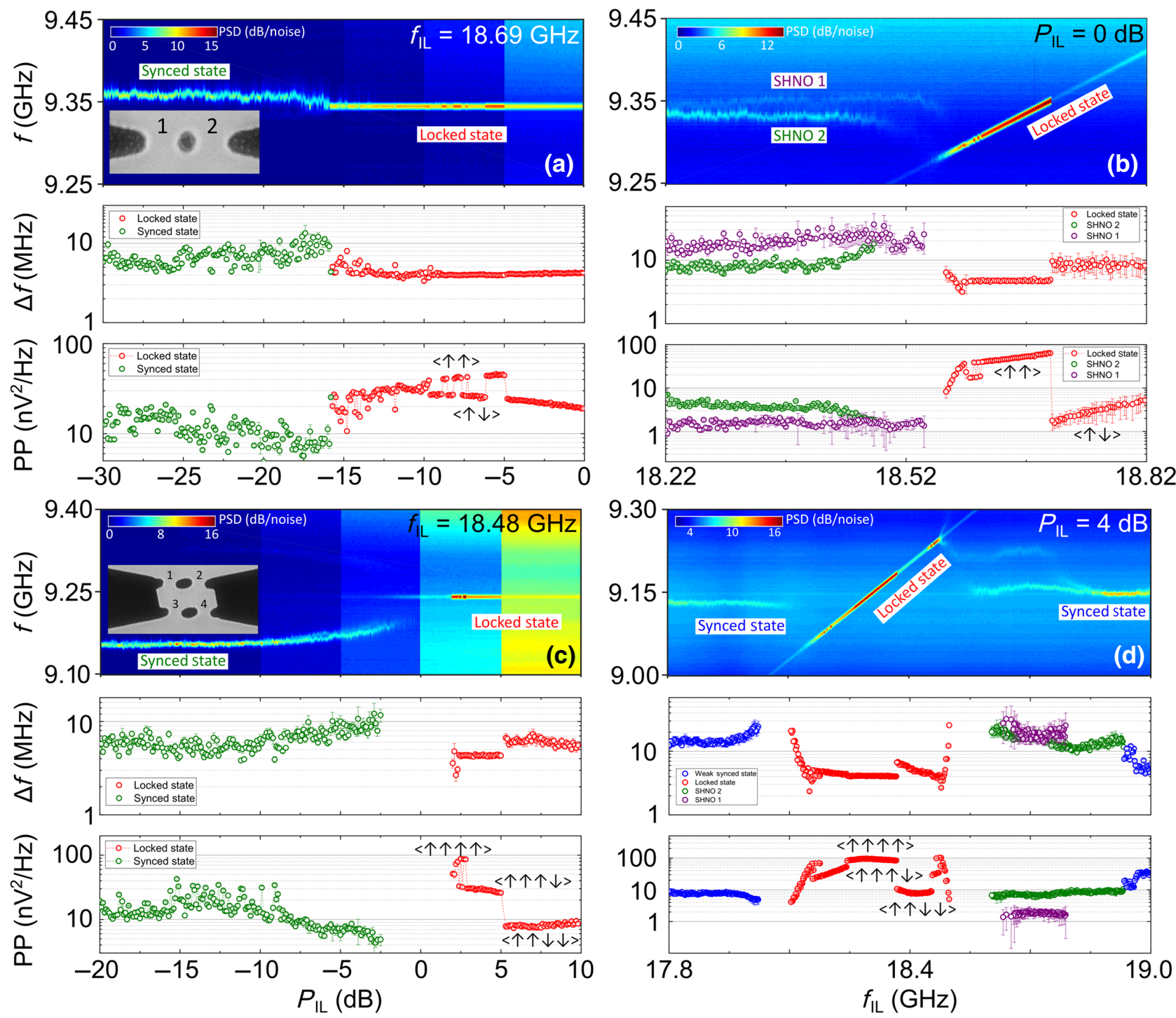

FIG. 2. Experimentally demonstrated phase binarization. PSD of a $1 \times 2$ SHNO array (a) vs injection power $P_{\mathrm{IL}}$ at a fixed injected frequency $f_{\mathrm{IL}}=18.69 \mathrm{GHz}$, and (b) vs $f_{\mathrm{IL}}$ at a fixed $P_{\mathrm{IL}}=0 \mathrm{~dB}$. In (a), the array starts off in a synchronized state ("Synced state"), while in (b) the SHNOs are not synchronized. Under SHIL, the linewidth decreases, while the power shows intermittent fluctuations between a high- and a low-power state. (c),(d) show the same trend for $2 \times 2$ arrays, where three different energy levels can be distinguished in the SHIL state. PP refers to peak power.

agreement with our simulations [45]. In particular, for an individual unbiased oscillator, there exist two degenerate injection-locked phase states. For two or more identical oscillators, in-phase or out-of-phase pairwise states are possible depending on the sign of the interoscillator coupling. It should be noted that, in the range of rf powers $[-15,-10] \mathrm{dBm}$, the SHIL and mutual synchronization compete, and no clear phase binarization is observed. With a further increase in $P_{\mathrm{IL}}$, phase binarization emerges as two distinct output-rf-power branches. Since the lower and higher output-power branches diverge with an increase in the injected rf strength, we conclude that the in-phase locked state, which we denote as $\langle\uparrow \uparrow\rangle$ from here on, increases in power (the relative phase difference decreases towards the ideal value of 0 ), and the out-of-phase state, $\langle\uparrow \downarrow\rangle$, decreases in power (the relative phase increases further towards the ideal value of $\pi$ ). Simultaneously, the energy landscape changes with the rf power, possibly due to heating, which affects the SHNOs individually as well as their couplings, and, as a result, the probability of ending up in a specific state also changes, in agreement with the slower hopping and final stability observed in Fig. 2(a). Since the system finally settles in the $\langle\uparrow \downarrow\rangle$ state, we conclude that the interoscillator coupling is negative.

We would like to point out that, for identical oscillators, pairwise phase states are doubly degenerate, i.e., the 
$\langle\uparrow \downarrow\rangle$ state is indistinguishable from $\langle\downarrow \uparrow\rangle$, and $\langle\uparrow \uparrow\rangle$ from $\langle\downarrow \downarrow\rangle$. The degeneracy can be lifted by applying oscillatorspecific biases by detuning the free-running frequencies from the common value of $f_{\mathrm{IL}} / 2$, e.g., with an applied dc current, owing to the large nonlinearity of SHNOs. Real devices might have intrinsic biases due to fabricationrelated differences in their free-running frequencies.

Fixing $P_{\mathrm{IL}}$ and sweeping $f_{\mathrm{IL}}$ instead reproduces qualitatively the same phase-binarization phenomenon, as shown in Fig. 2(b). In this annealing scheme, we coherently vary the biases of the oscillators, as is evident from linear term in Eq. (2). In this measurement, the two SHNOs are at first not mutually synchronized, and operate at slightly different intrinsic frequencies. However, as $f_{\mathrm{IL}}$ approaches the frequency of the SHNOs $\left(f_{\mathrm{SHNO}}\right)$, first one and then both of the SHNOs become locked, and, after a region of unstable fluctuations, the SHNOs first fall into the $\langle\uparrow \uparrow\rangle$ state and eventually switch into the $\langle\uparrow \downarrow\rangle$ state. It is noteworthy that the system switches from $\langle\uparrow \uparrow\rangle$ to $\langle\uparrow \downarrow\rangle$ when the injected frequency increases beyond the intrinsic frequencies of both oscillators instead of being in between them. This is again consistent with negative interoscillator coupling. In addition, the difference in peak power between the two states is now close to 2 orders of magnitude, indicating close to perfect cancellation of the microwave signal in the $\langle\uparrow \downarrow\rangle$ state.

We now turn to the two-dimensional $2 \times 2$ arrays, where similar phase binarization can be observed, with yet more phase binarized states appearing. As seen in Fig. 2(c), a similar trend to that for the $1 \times 2$ array can be observed, i.e., the four SHNOs start out in a mutually synchronized state, which becomes injection locked to the external signal at about $-2 \mathrm{dBm}$. Here, the microwave signal all but disappears, which we interpret as showing a complete antiphase state of the type $\langle\uparrow \uparrow \downarrow \downarrow\rangle$, or any of its equivalent degenerate states. In a tiny region around $2 \mathrm{dBm}$, the array switches into a high-power state, which we interpret as $\langle\uparrow \uparrow \uparrow \uparrow\rangle$. However, this high-power state is soon replaced by an intermediate state with about one quarter of the power, consistent with a $\langle\uparrow \uparrow \uparrow \downarrow\rangle$ state or any of its degenerate states. At $5 \mathrm{dBm}$, a very low-power antiphase state is again reached. Just as for the $1 \times 2$ array, the antiphase state is favored at high injected power.

To gain further insight into these different states and allow us to determine the exact phase of each nanoconstriction inside the arrays, we now turn to phase-BLS microscopy . Figures 3(a)-3(e) show the phase-BLS results from the $1 \times 2$ SHNO array biased at $I_{\mathrm{dc}}=2.8 \mathrm{~mA}$ in a magnetic field $H=5930 \mathrm{Oe}$, resulting in a free-running frequency $f_{\mathrm{SHNO}} \simeq 7.8 \mathrm{GHz}$. The array is under SHIL, with $P_{\mathrm{IL}}=0 \mathrm{dBm}$ and $f_{\mathrm{IL}}=15.6 \mathrm{GHz}$. Figure 3(a) shows an SEM image of the array, with dashed green arrows indicating the direction of a phase-BLS line scan across both nanoconstrictions. Figure 3(b) shows the phase-BLS counts vs location for the electrically determined $\langle\uparrow \downarrow\rangle$ state, where the phase-BLS detection angle is set to $90^{\circ}$. The line scan shows a high SW intensity in SHNO 1 only. When the phase-BLS detection angle is rotated by $180^{\circ}$ as in Fig. 3(b), the counts are instead located in SHNO 2. The electrically measured $\langle\uparrow \downarrow\rangle$ state can hence be directly mapped onto an antiphase state in the auto-oscillations of SHNOs 1 and 2. Similarly, when we change the experimental conditions slightly to realize an electrical $\langle\uparrow \uparrow\rangle$ state, the same type of phase-BLS line scans now clearly show in Figs. 3(d) and 3(e) that the two SHNOs are exactly in phase with each other.

Similar line scans can now be done for the $2 \times 2$ SHNO array, as shown in Fig. 3(f). In Figs. 3(g)-3(j), the array is supplied with $I_{\mathrm{dc}}=3 \mathrm{~mA}$ in a field $H=5980 \mathrm{Oe}$, and $P_{\mathrm{IL}}=+4 \mathrm{dBm}$ is used for SHIL; the detected electrical microwave signal is vanishingly small, indicating an antiphase state of the array. The two phase-BLS line scans directly corroborate this picture, as SHNOs 1 and 4 have a $0^{\circ}$ relative phase difference while the array operates with a $180^{\circ}$ phase difference in SHNOs 2 and 3. This arrangement corresponds to a $\langle\downarrow \uparrow \uparrow \downarrow\rangle$ phase state, which is reflected in a low-peak-power state in the electrical measurement. When we increase $H$ to $6000 \mathrm{Oe}$, we find from the electrical measurements that a fully in-phase $\langle\downarrow \downarrow \downarrow \downarrow\rangle$ state is favored. This is again directly corroborated by the phase-BLS scans in Figs. 3(k)-3(n).

In addition, we can also realize the intermediate electrical state and now directly observe which SHNO is out of phase with the others. Instead of line scans, we want to highlight here the sinusoidal phase dependence of the phase-BLS counts as a function of the relative injectionlocking phase angle. We measure the phase-BLS counts in four spatial locations, corresponding to each nanoconstriction center. On varying the phase angle from 0 to $360^{\circ}$, we clearly observe that SHNOs 1, 2, and 4 show the same type of sinusoidal dependence of the BLS counts vs phase angle, whereas SHNO 3 shows exactly the opposite behavior. In other words, phase-BLS allows us to determine the exact $\langle\downarrow \downarrow \uparrow \downarrow\rangle$ state out of the four degenerate possibilities for the intermediate state.

\section{DISCUSSION}

The $2 \times 2$ SHNO array that we present is an example of a nanoscale-oscillator-based IM that is able to minimize an Ising Hamiltonian. Here, we show that the phase patterns obtained from the phase-BLS measurements are indeed consistent solutions to max-cut problems defined by coupling and bias parameters.

For the simple $2 \times 2$ array that we discuss here, it is easy to enumerate all possible $2^{4}$ solutions to the given optimization problem and verify the result obtained from the BLS measurement. We therefore focus on simulating such an array. Micromagnetic simulations are performed using the graphics-processing-unit-accelerated program 

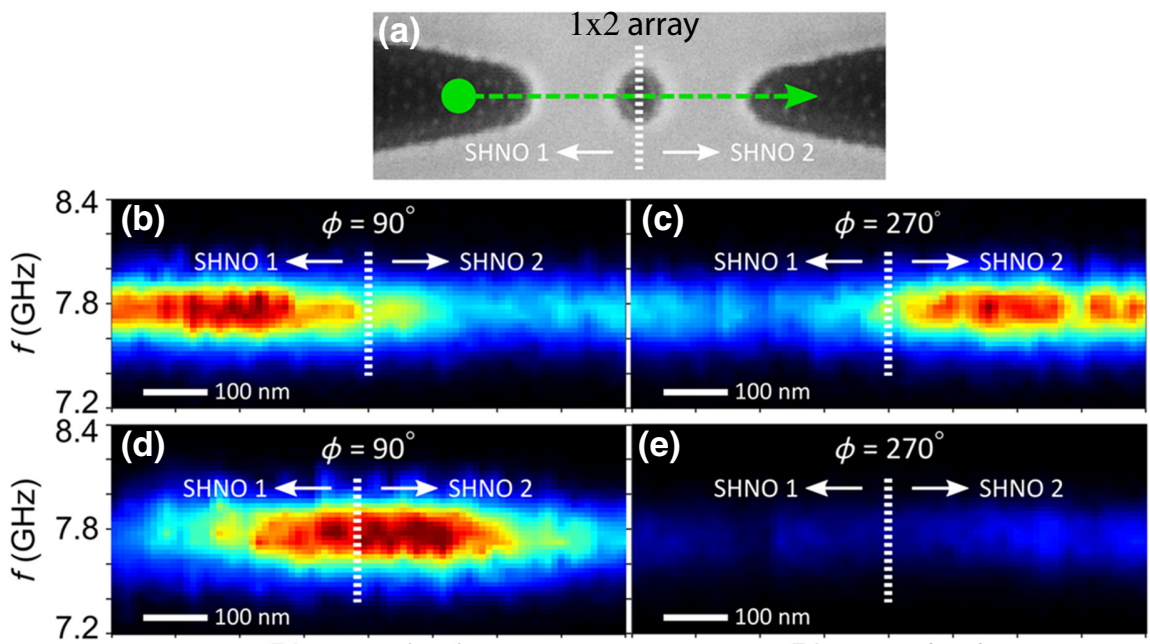

Distance $(\mathrm{nm})$

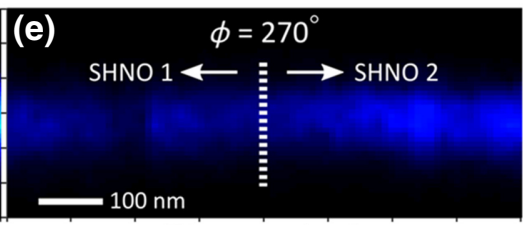

Distance $(\mathrm{nm})$
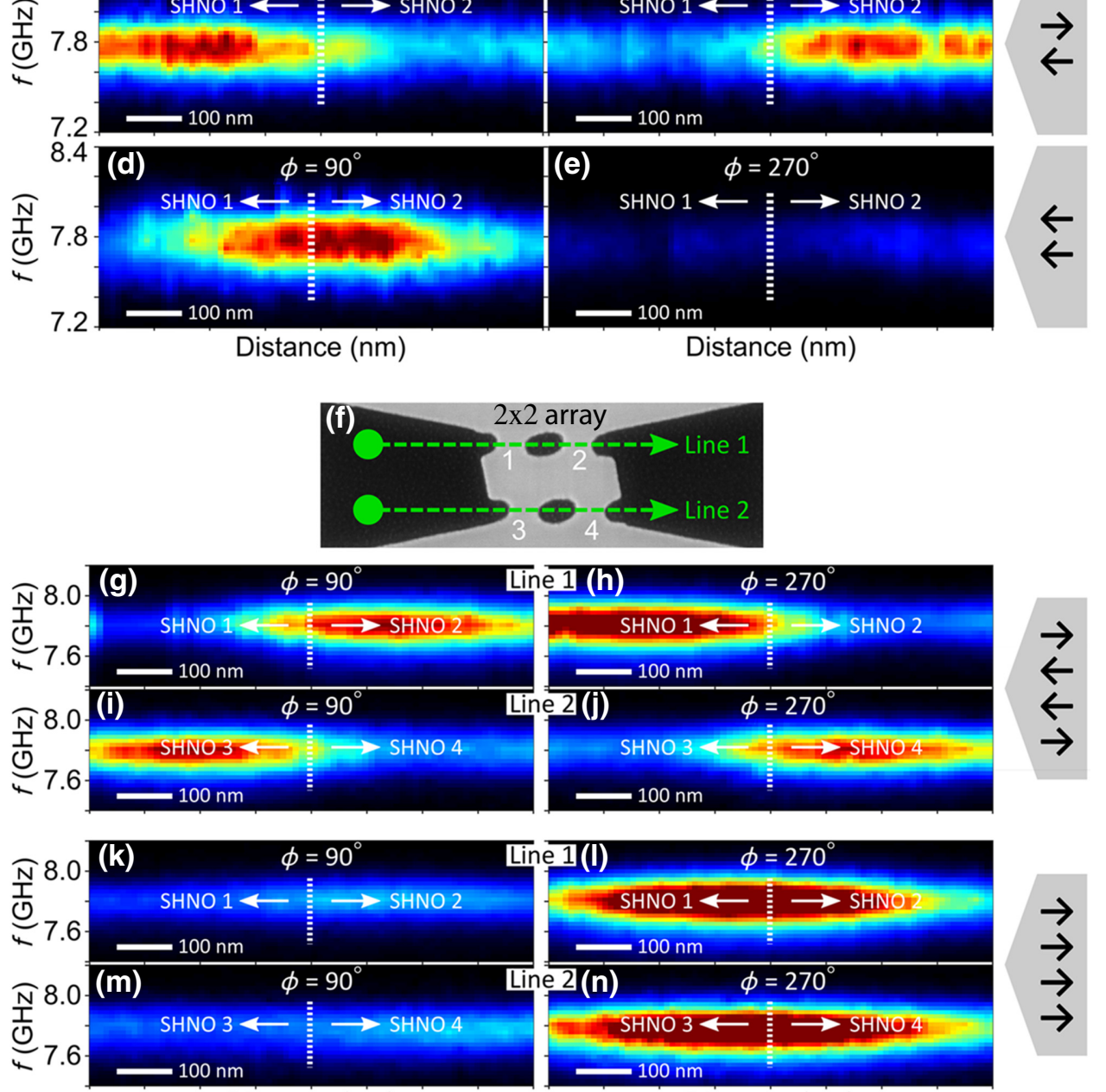

Distance $(\mathrm{nm})$

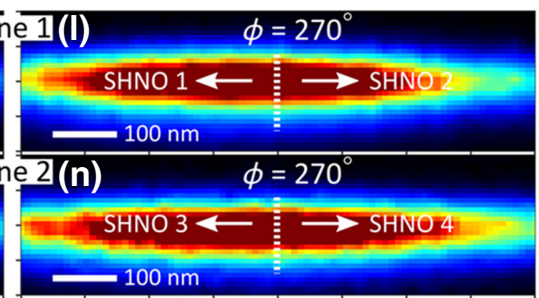

Distance (nm)
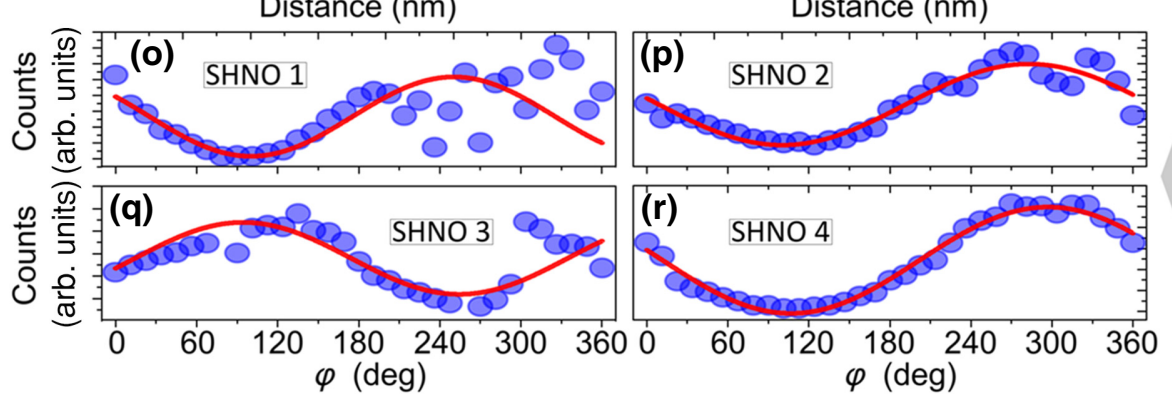

FIG. 3. Phase-resolved BLS microscopy of individual SHNOs. (a) SEM image of the $1 \times 2$ SHNO array under study; the green arrow shows the direction of the BLS line scan. SHIL with $P_{\mathrm{IL}}=0 \mathrm{dBm}$ and $f_{\mathrm{IL}}=15.6 \mathrm{GHz}$ is used. (b)-(e) show the spin wave (SW) intensity profile of the SHNOs for the phase-resolved BLS signal at $90^{\circ}$ and $270^{\circ}$. The SHNOs in (b),(c) are energized with a $180^{\circ}$ phase difference $(\langle\uparrow \downarrow\rangle)$ when $2 f_{\text {SHNO }}<f_{\text {IL }}$, and in (d),(e) with a $0^{\circ}$ phase difference $(\langle\uparrow \uparrow\rangle)$ when $2 f_{\text {SHNO }} \simeq f_{\mathrm{IL}}$. (f) SEM image of a $2 \times 2$ SHNO array with corresponding BLS line-scan directions. Phaseresolved BLS line scans are shown in $(\mathrm{g})-(\mathrm{j})$ for $P_{\mathrm{IL}}=+4$ $\mathrm{dBm}$, corresponding to the $\langle\downarrow \uparrow \uparrow \downarrow\rangle$ phase state, while (k)-(n) show the $\langle\downarrow \downarrow \downarrow \downarrow\rangle$ state obtained at $P_{\mathrm{IL}}=+3 \mathrm{dBm}$. (o)-(r) BLS counts for the least stable phase state $\langle\downarrow \downarrow \uparrow \downarrow\rangle$ at $P_{\mathrm{IL}}=-2 \mathrm{dBm}$, measured by sweeping the phase $(\varphi)$ of the BLS.
MuMax3 [46]. The system geometry is obtained from the computer-aided-design model used during the nanofabrication process and discretized into $1024 \times 1024 \times 1$ grid cells representing the $\mathrm{Ni}_{80} \mathrm{Fe}_{20}$ layer of the stack. The individual size of each cell is $4 \times 4 \times 3 \mathrm{~nm}$. The current and Oersted-field distributions are calculated separately and included in the simulation as a source of the spin current originating from the spin Hall effect and as an external magnetic field, respectively. The parameters used in the simulations are chosen to mimic the experimental conditions as closely as possible, including a saturation magnetization $\mu_{0} M_{S}=0.59 \mathrm{~T}$, a Gilbert damping constant $\alpha=0.025$, a spin Hall angle $\theta_{\mathrm{SH}}=0.09$, and an exchange constant $A_{\text {ex }}=10 \mathrm{pJ} / \mathrm{m}$. The magnetic field is applied at 
an angle of $78^{\circ}$ to the sample plane and a $24^{\circ}$ in-plane angle. Both the value of the dc current and the amplitude of the ac binarization current are equal to $3 \mathrm{~mA}$. Each simulation lasts $150 \mathrm{~ns}$, where the first $50 \mathrm{~ns}$ are used for initialization of the magnetization dynamics without ac excitation and the remaining 100 ns include the binarization current. FFT amplitude and phase diagrams are produced based on the last $10 \mathrm{~ns}$ of each simulation, using custom software [47]. We first recreate the measurement results using the method described above. After applying an external magnetic field with a magnitude of 5980 Oe and a rf current with a frequency of $15.6 \mathrm{GHz}$, we observe stable injection-locked oscillations at $7.8 \mathrm{GHz}$, which is close to the natural frequency of the system. We then

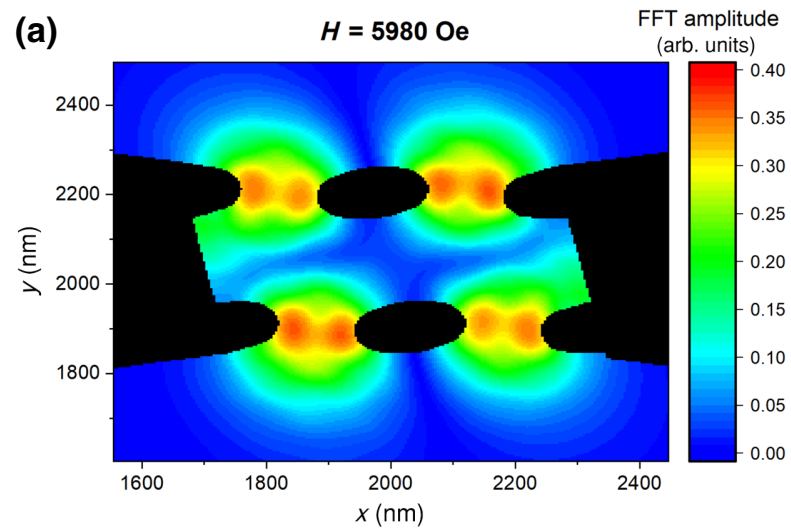

(c)

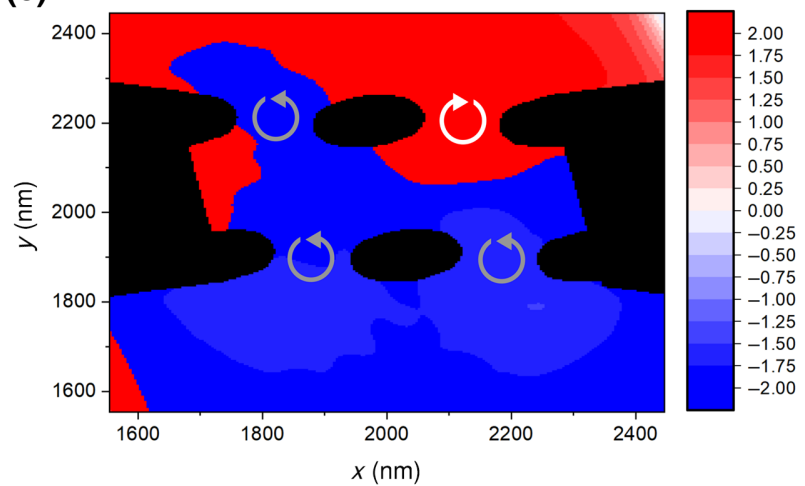

(e)

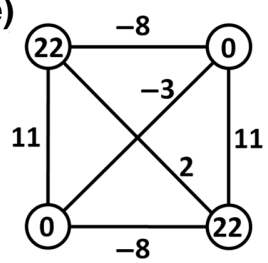

(f)

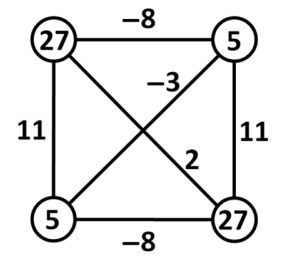

perform a pointwise FFT on the simulated data and obtain the spatially resolved amplitude and phase distributions of the auto-oscillation shown in Figs. 4(a) and 4(b), respectively. The amplitude map confirms that all four SHNOs auto-oscillate in the same type of mode, while one can see a clear antiparallel $\langle\downarrow \uparrow \uparrow \downarrow\rangle$ phase pattern (the lowestoutput-rf-power state) that is identical to the results of the phase-BLS measurements in Figs. 3(g)-3(j). A slight increase in the applied field to 5990 Oe favors an intermediate $\langle\downarrow \uparrow \downarrow \downarrow\rangle$ phase pattern [Fig. 4(c)], in agreement with Figs. 3(o)-3(r). Finally, increasing the field further to 6020 Oe yields a spatially uniform $\langle\downarrow \downarrow \downarrow \downarrow\rangle$ phase state (with the highest output rf power), as shown in Fig. 4(d), which is consistent with Figs. $3(\mathrm{k})-3(\mathrm{n})$. It is worth noting that in
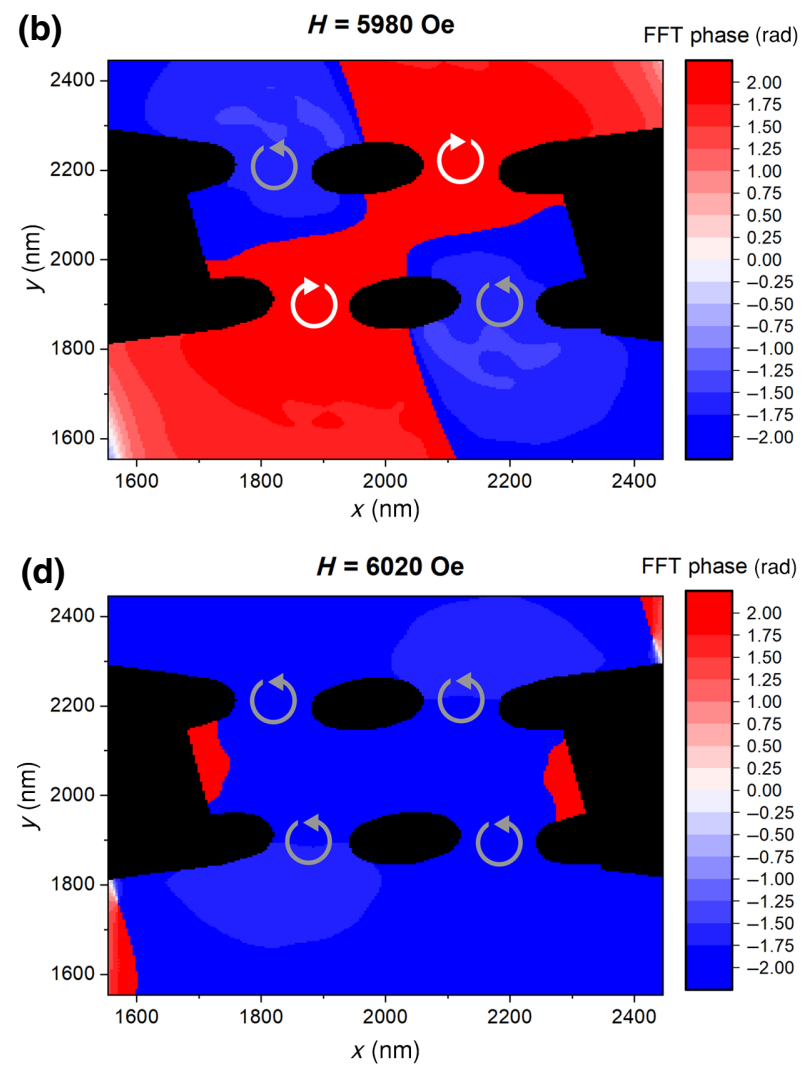

(g)

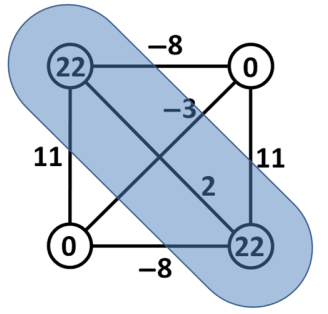

(h)

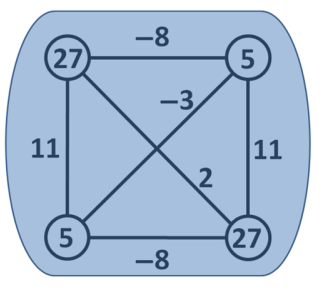

FIG. 4. Micromagnetic simulations of a $2 \times 2$ SHNO Ising machine. (a),(b) Spatial maps of the FFT amplitude and phase, respectively, obtained for a 7.8-GHz mode under a field $H=5980$ Oe. Phase maps are also shown for $H$ increased to 5990 Oe (c) and to $6020 \mathrm{Oe}(\mathrm{d})$, where the change in the Ising-problem parameters results in different phase patterns. The modified max-cut problems corresponding to fields of 5980 Oe (e) and 6000 Oe (f) are also shown. By reading out the phase patterns of the SHNO network, optimal solutions to these problems are obtained, as illustrated in $(\mathrm{g}),(\mathrm{h})$. 
the current experiments, we do not optimize or implement an annealing scheme that ensures the optimal solution. For the optimal solution to be obtained, an adiabatic problemspecific annealing scheme needs to be implemented, which is beyond the scope of this study.

Since the phase states of the SHNO array change with the applied magnetic field, we conclude that the parameters of the corresponding Ising Hamiltonian, i.e., the couplings and biases, are field-tunable. While this paves the way for on-chip IM control, e.g., with an Oersted field produced by microstrips placed next to each SHNO, we believe that the recently demonstrated voltage control of SHNOs will be a superior alternative [31].

To figure out which particular CO problem our IM solves, we employ a semiempirical method to estimate the coupling and bias coefficients based on the final phase state of the system, as explained in Sec. II. We eventually arrive at a modified version of the well-known max-cut problem [48] given by the graphs presented in Figs. 4(e) and 4(f), which correspond to $H=5980$ Oe and $H=$ 6000 Oe, respectively. Each graph has weighted edges (denoted by numbers next to the edges) and also an additional weight on top of each vertex (denoted by numbers in circles), corresponding to the coupling and bias terms in QUBO notation. We immediately observe that a 20 Oe change in the applied field leads to significant change in the bias terms. It should be noted that, in the present architecture, the auto-oscillations are confined to the constrictions [49], and the coupling is mediated by wireless instantaneous magnetodipolar interactions. While the coupling is in principle tunable with the applied magnetic field and bias current [see Eq. (A2)], it is not straightforward to change it without also altering the bias terms. Future studies could potentially make use of spin-wave-mediated coupling, which should be stronger, possible to timedelay, and independently controllable with voltage gating [33,50-52]. We also would like to highlight that oscillators from different columns are negatively coupled, consistent with our experimental results on $1 \times 2$ arrays.

The task of the IM is now to identify the optimal subset of each graph in such a way that the sum of weights on the edges being cut and on the vertices being covered has a maximum. The solutions, obtained in the form of the $\langle\downarrow \uparrow \uparrow \downarrow\rangle$ state and $\langle\downarrow \downarrow \downarrow \downarrow\rangle$ state as discussed previously, are illustrated in Figs. 4(g) and 4(h). In the first case, the optimal subset incorporates two opposite vertices, with most edges being cut. In the second case, a small change in the vertex weights leads to a different situation, where the optimal solution includes the entire original graph, and no edges are cut. This happens due to the fact that cutting off any vertex in the second graph would result in a lower sum of weights and thus a nonoptimal solution to the max-cut problem.

For a direct comparison with a state-of-the-art Ising machine, we also solve the very same max-cut problem on the D-Wave Advantage ${ }^{\mathrm{TM}}$ system using D-Wave's Leap ${ }^{\mathrm{TM}}$ quantum cloud service [53]. We rely on a quantum sampler with automatic embedding. Sampling ten times is sufficient to obtain correct solutions for the graphs shown in Figs. 4(e) and 4(f), with an average sampling time of $81 \mu \mathrm{s}$ per sample, including annealing for $20 \mu \mathrm{s}$, followed by approximately $40 \mu$ s for readout and $20 \mu$ s for cooldown, as summarized in Table I. It is worth mentioning that the total time to solution is around $18 \mathrm{~ms}$, including approximately $17 \mathrm{~ms}$ of programming time and $0.3 \mathrm{~ms}$ of postprocessing time. However, we deliberately do not include those in our calculations, as (i) they would be negligible compared with the sampling times required for large dense problems, and (ii) one might expect them to be substantially reduced as the technology moves forward.

Assuming the publicly advertised maximum power consumption of D-Wave Advantage ${ }^{\mathrm{TM}}$ of $25 \mathrm{~kW}$, we conclude that the energy consumption of the system is approximately $2 \mathrm{~J}$ per sample, excluding the preprocessing and postprocessing stages. According to D-Wave, most of this energy goes into the cooling system and is hence consumed continuously.

Although we do not have firm performance numbers for our proof-of-principle IM architecture, we make some conservative performance estimates here and compare them with D-Wave's metrics. First of all, our simulations and experiments show that the relaxation rate of a SHNO towards stable auto-oscillations is on the order of $2 \pi / \alpha \omega_{0} \approx 5$ ns, i.e., 3 orders of magnitude shorter than the annealing time for $\mathrm{D}$-Wave Advantage ${ }^{\mathrm{TM}}$; here, $\alpha \approx 0.022$ is the Gilbert damping constant, and $\omega_{0} \approx 2 \pi \times 9.48 \mathrm{GHz}$ is the SHNO auto-oscillation frequency. Although the relaxation time of an array is expected to increase with the number of oscillators $N$ [54], it should be possible to achieve approximately $\log N$ scaling by means of a corresponding design of the coupling strength [55], which should provide substantially good performance even for quite large networks of SHNOs. Simulations demonstrate that comparable spin-torque nano-oscillators (STNOs) can

TABLE I. Performance and energy-efficiency comparison.

\begin{tabular}{lcccccc}
\hline \hline & $\begin{array}{c}\text { Annealing } \\
\text { time }(\mu \mathrm{s})\end{array}$ & $\begin{array}{c}\text { Readout } \\
\text { time }(\mu \mathrm{s})\end{array}$ & $\begin{array}{c}\text { Settling } \\
\text { time }(\mu \mathrm{s})\end{array}$ & $\begin{array}{c}\text { Sampling } \\
\text { time }(\mu \mathrm{s})\end{array}$ & $\begin{array}{c}\text { Power } \\
\text { usage }(\mathrm{W})\end{array}$ & $\begin{array}{c}\text { Energy per } \\
\text { sample }(\mathrm{J})\end{array}$ \\
\hline $\begin{array}{l}\text { D-Wave Advantage } \\
\text { 5M }\end{array}$ & 20 & $\approx 40$ & 21 & 81 & 25000 & $\approx 2$ \\
\hline \hline
\end{tabular}


anneal a max-cut problem with 288 variables in around 450 ns with a probability of $50 \%$ of achieving an optimal solution in 100 samples [45]. Moreover in Ref. [56], a simulated array of 2500 STNOs was able to fully synchronize on a timescale of around $1.5 \mu$ s even without a sophisticated annealing scheme. Therefore, we are confident that large-scale SHNO arrays could perform adiabatic annealing at least on a par with the time for D-Wave Advantage $^{\mathrm{TM}}$, which is generally problem-specific [17] but defaults to $20 \mu \mathrm{s}$. We then ultimately aim for onchip real-time measurements of the phases of the SHNOs. Assuming that the electronic front end, including lownoise amplifiers, current sources, and digital logic, works at the same frequencies as the SHNOs, e.g., by using a silicon-on-insulator process, we would expect a readout time of $2 \pi / \omega_{0}=100 \mathrm{ps}$. Now, for the power consumption per SHNO, we take into account a value for the Joule heating of a SHNO $P_{\mathrm{SHNO}}=I_{\text {bias }}^{2} R_{\mathrm{SHNO}}=2.25 \mathrm{~mW}$, where $I_{\text {bias }}=3 \mathrm{~mA}$ is the bias current and $R_{\mathrm{SHNO}} \approx 250 \Omega$ is the typical dc resistance of a $120-\mathrm{nm}$ SHNO, and an injected rf power of around $1 \mathrm{~mW}$, giving $3.25 \mathrm{~mW}$ in total. If we assume that the on-chip electronic front end consumes an order of magnitude more than the SHNO itself does, we arrive at a power consumption per "spin" of around $36 \mathrm{~mW}$. Assuming that the dc and rf power consumptions scale linearly with the number of oscillators in the array, we expect $180 \mathrm{~W}$ consumption for a system of 5000 "spins." This is within the range of the current generation of Peltier elements, and so we expect the postreadout cooling to be as fast as the spin-lattice relaxation time, which typically lies in the picosecond to nanosecond range. So, we finally arrive at a sampling time of $21 \mu$ s and an energy consumption of $0.0038 \mathrm{~J}$ per sample. While this number is already about 500 times smaller than that for the $\mathrm{D}$-Wave Advantage ${ }^{\mathrm{TM}}$ system, there is significant further potential for an additional 1-2 orders of magnitude lower energy consumption via both a reduction of the SHNO threshold current and an increase in the SHNO operational frequency $[30,31,57]$. It is also worth pointing out that our SHNO architecture consumes energy only on demand, i.e., during sampling, since it does not require an alwayson cryogenic cooling system. We therefore believe that, compared with D-Wave's Advantage ${ }^{\mathrm{TM}}$ system, our architecture holds significant promise for substantially reduced power consumption, faster sampling, and a substantially smaller footprint.

As SHNO arrays as large as $10 \times 10$ have been shown to be fully operational with strong interoscillator coupling, we argue that it should be possible to scale up our present demonstration to very large arrays. We also believe that voltage-controlled magnetic anisotropy in Co-Fe-Bbased SHNOs [33] could be a possible route towards a fully integrated voltage-controlled SHNO Ising machine. For fast electrical readout, one could also add magnetic tunnel junctions to the auto-oscillating regions of each nanoconstriction. While these are important challenges, the very high operating frequency, the very high degree of miniaturization, the demonstrated scaling path, and the CMOS compatibility all hold tremendous promise for a groundbreaking technology platform aimed towards widepurpose Ising machines.

\section{ACKNOWLEDGMENTS}

This work was partially supported by the Horizon 2020 research and innovation program (ERC Advanced Grant No. 835068, "TOPSPIN"). This work was also partially supported by the Swedish Research Council (VR) and the Knut and Alice Wallenberg Foundation. J.Ch. acknowledges funding from Program No. 2017/24/T/ST3/00009 of the National Science Center, Poland. The numerical calculations were supported in part by the PL-Grid infrastructure. We acknowledge D-Wave Systems Inc. for providing developers, researchers, and academics with access to quantum computing technology via their cloud service Leap $^{\mathrm{TM}}$.

\section{APPENDIX: ISING-HAMILTONIAN CALCULATIONS AND MODIFIED MAX-CUT PROBLEM}

Based on the results of the micromagnetic simulations, we can expect the oscillation mode in the $\mathrm{Pt} / \mathrm{Ni}_{80} \mathrm{Fe}_{20}$ bilayer to be localized near the constriction area. Therefore, we neglect the exchange-mediated contribution to the coupling and focus only on the magnetodipolar contribution. Assuming that all oscillators in the array have identical physical parameters and taking into account the vectorial structure of the precession in the applied field [50,58], we expect that the coupling strength $J_{i j}$ between the $i$ th and $j$ th oscillators will be given by

$$
\begin{aligned}
J_{i j}= & \sqrt{1+v^{2}} \frac{V_{\text {eff }}}{a_{i j}{ }^{3}} \frac{\gamma^{2} M_{S}}{2 \omega_{0}}\left[H\left(3 \sin ^{2} \theta \cos ^{2} \phi_{i j}-1\right)\right. \\
& \left.+M_{S} \sin ^{2} \theta\left(1-3 \sin ^{2} \phi_{i j}\right)\right] .
\end{aligned}
$$

Here, $v$ is the normalized nonlinear frequency-shift coefficient, $V_{\text {eff }}$ is the effective oscillator volume, $a_{i j}$ is the distance between two oscillators, $\gamma$ is the gyromagnetic ratio, $\omega_{0}$ is the generated frequency, $\theta$ is the angle between the external magnetic field $H$ and the sample plane, and $\phi_{i j}$ is the angle between the in-plane component of the external magnetic field and the line connecting the $i$ th and $j$ th oscillators. For the purpose of Ising-machine calculations, it is convenient to introduce a constant factor $J_{0}$ that is approximately the same for all oscillators in the array:

$$
J_{0}=\sqrt{1+v^{2}} \frac{V_{\text {eff }}}{a_{0}{ }^{3}} \frac{\gamma^{2} M_{S}}{2 \omega_{0}} H,
$$


where $a_{0}$ is the pitch size of the array, equal to $300 \mathrm{~nm}$. This allows us to express the individual $J_{i j}$ values as

$$
\begin{aligned}
J_{i j}= & J_{0}\left(\frac{a_{0}}{a_{i j}}\right)^{3}\left(3 \sin ^{2} \theta \cos ^{2} \phi_{i j}-1\right. \\
& \left.+\frac{M_{S}}{H} \sin ^{2} \theta\left(1-3 \sin ^{2} \phi_{i j}\right)\right) .
\end{aligned}
$$

For the parameters used in our system, we expect $J_{0}$ to be of the order of magnitude of 50-200 MHz, which is also consistent with our micromagnetic simulations. In order to obtain the best fit with the experiment, from now on we assume $J_{0}=87 \mathrm{MHz}$. To calculate the values of $J_{i j}$, we now take into account the nonsymmetrical geometry of our system, and find that $a_{12}=a_{34}=300 \mathrm{~nm}$, $a_{13}=a_{24} \approx 305 \mathrm{~nm}, a_{23} \approx 385 \mathrm{~nm}, a_{14} \approx 467 \mathrm{~nm}, \phi_{12}=$ $\phi_{34}=24^{\circ}, \phi_{13}=\phi_{24}=77^{\circ}, \phi_{23}=27^{\circ}$, and $\phi_{14}=117^{\circ}$. From this, we calculate $J_{12}=J_{34}=1.87 J_{0}, J_{13}=J_{24}=$ $-2.47 J_{0}, J_{23}=0.77 J_{0}$, and $J_{14}=-0.46 J_{0}$.

As a result, the Lyapunov function minimized by the SHNO array [19] can be written as

$$
E(\vec{\phi})=-\sum_{i=1}^{N} \sum_{j \neq i}^{N} \tilde{J}_{i j} \cos \left(\phi_{i}-\phi_{j}\right)-\sum_{i=1}^{N} \frac{2 f_{i}-f_{\mathrm{inj}}}{f_{i}} \phi_{i} .
$$

Here, we neglect the binarization term for simplicity, since we focus on states that are already binarized, and introduce dimensionless coupling coefficients $\tilde{J}_{i j}=K J_{i j}$, where $K$ is an arbitrary scaling factor having units of time. Under the working conditions of our system, the oscillator phases are locked by the injected rf signal, which allows us to rewrite the previous equation in a more practical QUBO form, assuming $\phi_{i}=\pi x_{i}$ and $\cos \pi x_{i}=1-2 x_{i}$ :

$$
\begin{aligned}
E(\vec{x})= & -\sum_{i=1}^{N} \sum_{j \neq i}^{N} 4 \tilde{J}_{i j} x_{i} x_{j} \\
& +\sum_{i=1}^{N}\left[\sum_{j \neq i}^{N} 2 \tilde{J}_{i j}-\frac{\pi\left(2 f_{i}-f_{\text {inj }}\right)}{f_{i}}\right] x_{i},
\end{aligned}
$$

where constant terms are omitted and the $x_{i} \in 0,1$ are variables in the binary optimization problem. The SHNObased Ising machine works by minimizing the expression in Eq. (A5) in such a way that the resulting oscillator phases, when equal to 0 or $\pi$, correspond to the variables $x_{i}$ being equal to either 0 or 1 .

We also perform a set of additional micromagnetic simulations with no spin Hall effect present and find that, due to the geometrical asymmetry of the array, the effective field in the area of nanoconstrictions 1 and 4 is higher than the effective field in the area of nanoconstrictions 2 and 3 by approximately 50 Oe. We assume that this difference persists under spin-Hall-excitation conditions and leads to a difference in free-running frequencies between oscillators 1 and 4 on the one hand and oscillators 2 and 3 on the other equal to approximately $140 \mathrm{MHz}$. After supplying the array with $3 \mathrm{~mA}$ of dc current but no ac current, we find the frequency of the entire array to be 7.87 $\mathrm{GHz}$ for $H=5980 \mathrm{Oe}$ and $7.90 \mathrm{GHz}$ for $H=6000 \mathrm{Oe}$. Therefore, we assume that the values of $f_{i}$ in Eq. (A5) are $f_{1}=f_{4}=7.80 \mathrm{GHz}$ and $f_{2}=f_{3}=7.94 \mathrm{GHz}$ for $H=5980$ Oe, and $f_{1}=f_{4}=7.83 \mathrm{GHz}$ and $f_{2}=f_{3}=7.97 \mathrm{GHz}$ for $H=6000 \mathrm{Oe}$. The frequency of the injected binarization current remains the same in both cases and is equal to $f_{\text {inj }}=15.60 \mathrm{GHz}$. As the external magnetic field amplitude increases, it can potentially affect not only the values of $f_{i}$ but also those of $\tilde{J}_{i j}$. We calculate this effect using the same assumptions for $J_{0}$ as previously and find that it remains negligible compared with the changes in $f_{i}$, which is why we do not include it in the analysis.

To obtain a formulation of a max-cut problem for our system, we rewrite Eq. (A5) as

$$
E(\vec{x})=\sum_{i=1}^{N} \sum_{j \neq i}^{N} w_{i j}\left(2 x_{i} x_{j}-x_{i}-x_{j}\right)-\sum_{i=1}^{N} o_{i} x_{i}
$$

where $w_{i j}=-2 \tilde{J}_{i j}$ and $o_{i}=\pi\left(2 f_{i}-f_{\text {inj }}\right) / f_{i}$. The above term describes the Lyapunov function that is minimized by the Ising machine. Since max-cut problems are typically formulated in terms of maximization and not minimization, we replace the expression above with an expression that the Ising machine will maximize:

$$
\tilde{E}(\vec{x})=-E(\vec{x})=\sum_{i=1}^{N} \sum_{j \neq i}^{N} w_{i j}\left(x_{i}+x_{j}-2 x_{i} x_{j}\right)+\sum_{i=1}^{N} o_{i} x_{i} .
$$

Now, we notice that the term $x_{i}+x_{j}-2 x_{i} x_{j}$ describes the edge-cutting procedure in the classical max-cut problem [48]. If $x_{i}=x_{j}$ (both vertices are in the same subset) then the term vanishes, and if $x_{i} \neq x_{j}$ (the vertices belong to opposite subsets, i.e., the edge between them is cut) then the term is equal to 1 . Therefore, we can use this expression to evaluate possible subset cuts in a weighted graph with the $w_{i j}$ serving as weights. Additionally, the expression in Eq. (A7) includes a term that punishes (or rewards, depending on the sign of $o_{i}$ ) a proposed way of cutting for assigning a particular vertex to the subset denoted by $x_{i}$ equal to 1 . We also note here that, for the sake of convenience, the $w_{i j}$ and $o_{i}$ values shown in Figs. 4(e)-4(h) are rounded and rescaled by a constant factor of 200, which does not affect the maximization procedure. 
[1] E. Lawler, Combinatorial Optimization - Networks and Matroids (Holt, Rinehart and Winston, New York, 1976).

[2] F. Glover, G. Kochenberger, and Y. Du, A tutorial on formulating and using QUBO models (2019).

[3] A. Lucas, Ising formulations of many NP problems, Front. Phys. 2, 5 (2014).

[4] S. Jiang, K. A. Britt, A. J. McCaskey, T. S. Humble, and S. Kais, Quantum annealing for prime factorization, Sci. Rep. 8, 17667 (2018).

[5] B. Wang, F. Hu, H. Yao, and C. Wang, Prime factorization algorithm based on parameter optimization of ising model, Sci. Rep. 10, 7106 (2020).

[6] E. Farhi, J. Goldstone, S. Gutmann, J. Lapan, A. Lundgren, and D. Preda, A quantum adiabatic evolution algorithm applied to random instances of an NP-complete problem, Science 292, 472 (2001).

[7] S. Boixo, T. F. Rønnow, S. V. Isakov, Z. Wang, D. Wecker, D. A. Lidar, J. M. Martinis, and M. Troyer, Evidence for quantum annealing with more than one hundred qubits, Nat. Phys. 10, 218 (2014).

[8] S. Tsukamoto, M. Takatsu, S. Matsubara, and H. Tamura, An accelerator architecture for combinatorial optimization problems, Fujitsu Sci. Tech. J. 53, 8 (2017).

[9] M. Yamaoka, C. Yoshimura, M. Hayashi, T. Okuyama, H. Aoki, and H. Mizuno, in 2015 IEEE International SolidState Circuits Conference-(ISSCC) Digest of Technical Papers (IEEE, 2015), p. 1.

[10] D. Oku, K. Terada, M. Hayashi, M. Yamaoka, S. Tanaka, and N. Togawa, A fully-connected ising model embedding method and its evaluation for CMOS annealing machines, IEICE Trans. Inf. Syst. 102, 1696 (2019).

[11] B. Sutton, K. Y. Camsari, B. Behin-Aein, and S. Datta, Intrinsic optimization using stochastic nanomagnets, Sci. Rep. 7, 44370 (2017).

[12] A. Parihar, N. Shukla, M. Jerry, S. Datta, and A. Raychowdhury, Vertex coloring of graphs via phase dynamics of coupled oscillatory networks, Sci. Rep. 7, 911 (2017).

[13] J. Chou, S. Bramhavar, S. Ghosh, and W. Herzog, Analog coupled oscillator based weighted ising machine, Sci. Rep. 9, 1 (2019).

[14] C. Roques-carmes, Y. Shen, L. Jing, T. Dubcek, S. A. Skirlo, H. Bagherianlemraski, and M. Soljacic, Optical Ising machines and optical convolutional neural networks (2019), uS Patent App. 16/032, 737.

[15] Y. Yamamoto, K. Aihara, T. Leleu, K.-i. Kawarabayashi, S. Kako, M. Fejer, K. Inoue, and H. Takesue, Coherent ising machines: Optical neural networks operating at the quantum limit, Npj Quantum Inf. 3, 49 (2017).

[16] A. Marandi, Z. Wang, K. Takata, R. L. Byer, and Y. Yamamoto, Network of time-multiplexed optical parametric oscillators as a coherent ising machine, Nat. Photonics 8, 937 (2014).

[17] R. Hamerly, T. Inagaki, P. L. McMahon, D. Venturelli, A. Marandi, T. Onodera, E. Ng, C. Langrock, K. Inaba, and T. Honjo et al., Experimental investigation of performance differences between coherent Ising machines and a quantum annealer, Sci. Adv. 5, eaau0823 (2019).

[18] J. Boyd, Silicon chip delivers quantum speeds [news], IEEE Spectr. 55, 10 (2018).
[19] T. Wang and J. Roychowdhury, in International Conference on Unconventional Computation and Natural Computation (Springer, 2019), p. 232.

[20] A. M. Lyapunov, The general problem of the stability of motion, Int. J. Control. 55, 531 (1992).

[21] R. Adler, A study of locking phenomena in oscillators, Proceedings of the IRE 34, 351 (1946).

[22] P. Bhansali and J. Roychowdhury, in 2009 Asia and South Pacific Design Automation Conference (2009), p. 522.

[23] A. Neogy and J. Roychowdhury, in 2012 Design, Automation Test in Europe Conference Exhibition (DATE) (2012), p. 1209.

[24] T. Wang, L. Wu, and J. Roychowdhury, in Proceedings of the 56th Annual Design Automation Conference 2019 (2019), p. 1.

[25] V. E. Demidov, S. Urazhdin, A. Zholud, A. V. Sadovnikov, and S. O. Demokritov, Nanoconstriction-based spin-hall nano-oscillator, Appl. Phys. Lett. 105, 172410 (2014).

[26] M. Ranjbar, P. Dürrenfeld, M. Haidar, E. Iacocca, M. Balinskiy, T. Le, M. Fazlali, A. Houshang, A. Awad, R. Dumas, and J. Åkerman, CoFeB-based spin Hall nano-oscillators, IEEE Magn. Lett. 5, 3000504 (2014).

[27] T. Chen, R. K. Dumas, A. Eklund, P. K. Muduli, A. Houshang, A. A. Awad, P. Dürrenfeld, B. G. Malm, A. Rusu, and J. Åkerman, Spin-torque and spin-hall nanooscillators, Proc. IEEE 104, 1919 (2016).

[28] R. H. Liu, W. L. Lim, and S. Urazhdin, Spectral Characteristics of the Microwave Emission by the Spin Hall Nano-Oscillator, Phys. Rev. Lett. 110, 147601 (2013).

[29] V. E. Demidov, S. Urazhdin, H. Ulrichs, V. Tiberkevich, A. Slavin, D. Baither, G. Schmitz, and S. O. Demokritov, Magnetic nano-oscillator driven by pure spin current, Nat. Mater. 11, 1028 (2012).

[30] M. Zahedinejad, H. Mazraati, H. Fulara, J. Yue, S. Jiang, A. A. Awad, and J. Åkerman, CMOS compatible $\mathrm{W} / \mathrm{CoFeB} / \mathrm{MgO}$ spin Hall nano-oscillators with wide frequency tunability, Appl. Phys. Lett. 112, 132404 (2018).

[31] H. Fulara, M. Zahedinejad, R. Khymyn, M. Dvornik, S. Fukami, S. Kanai, H. Ohno, and J. Åkerman, Giant voltage-controlled modulation of spin Hall nano-oscillator damping, Nat. Commun. 11, 4006 (2020).

[32] M. Haidar, A. A. Awad, M. Dvornik, R. Khymyn, A. Houshang, and J. Åkerman, A single layer spin-orbit torque nano-oscillator, Nat. Commun. 10, 2362 (2019).

[33] H. Fulara, M. Zahedinejad, R. Khymyn, A. A. Awad, S. Muralidhar, M. Dvornik, and J. Åkerman, Spinorbit torque-driven propagating spin waves, Sci. Adv. 5, eaax8467 (2019).

[34] A. Awad, A. Houshang, M. Zahedinejad, R. Khymyn, and J. Åkerman, Width dependent auto-oscillating properties of constriction based spin Hall nano-oscillators, Appl. Phys. Lett. 116, 232401 (2020).

[35] H. Mazraati, M. Zahedinejad, and J. Åkerman, Improving the magnetodynamical properties of $\mathrm{NiFe} / \mathrm{Pt}$ bilayers through Hf dusting, Appl. Phys. Lett. 113, 092401 (2018).

[36] M. Zahedinejad, A. A. Awad, P. Dürrenfeld, A. Houshang, Y. Yin, P. K. Muduli, and J. Åkerman, Current modulation of nanoconstriction spin-hall nano-oscillators, IEEE Magn. Lett. 8, 3704804 (2017). 
[37] T. Kendziorczyk and T. Kuhn, Mutual synchronization of nanoconstriction-based spin Hall nano-oscillators through evanescent and propagating spin waves, Phys. Rev. B 93, 134413 (2016).

[38] A. A. Awad, P. Dürrenfeld, A. Houshang, M. Dvornik, E. Iacocca, R. K. Dumas, and J. Åkerman, Long-range mutual synchronization of spin Hall nano-oscillators, Nat. Phys. 13, 292 (2017).

[39] V. E. Demidov, H. Ulrichs, S. V. Gurevich, S. O. Demokritov, V. S. Tiberkevich, A. N. Slavin, A. Zholud, and S. Urazhdin, Synchronization of spin Hall nano-oscillators to external microwave signals, Nat. Commun. 5, 3179 (2014).

[40] T. Hache, T. Weinhold, K. Schultheiss, J. Stigloher, F. Vilsmeier, C. Back, S. S. Arekapudi, O. Hellwig, J. Fassbender, and H. Schultheiss, Combined frequency and time domain measurements on injection-locked, constrictionbased spin Hall nano-oscillators, Appl. Phys. Lett. 114, 102403 (2019).

[41] L. Yang, R. Verba, V. Tiberkevich, T. Schneider, A. Smith, Z. Duan, B. Youngblood, K. Lenz, J. Lindner, A. N. Slavin, and I. N. Krivorotov, Reduction of phase noise in nanowire spin orbit torque oscillators, Sci. Rep. 5, 16942 (2015).

[42] C. Roques-Carmes, Y. Shen, C. Zanoci, M. Prabhu, F. Atieh, L. Jing, T. Dubček, C. Mao, M. R. Johnson, V. Čeperić, J. D. Joannopoulos, D. Englund, and M. Soljačić, Heuristic recurrent algorithms for photonic ising machines, Nat. Commun. 11, 249 (2020).

[43] P. Dürrenfeld, A. A. Awad, A. Houshang, R. K. Dumas, and J. Åkerman, A $20 \mathrm{~nm}$ spin Hall nano-oscillator, Nanoscale 9, 1285 (2017).

[44] M. Zahedinejad, A. A. Awad, S. Muralidhar, R. Khymyn, H. Fulara, H. Mazraati, M. Dvornik, and J. Åkerman, Two-dimensional mutually synchronized spin Hall nanooscillator arrays for neuromorphic computing, Nat. Nanotech. 15, 47 (2020).

[45] D. I. Albertsson, M. Zahedinejad, A. Houshang, R. Khymyn, J. Åkerman, and A. Rusu, Ultrafast Ising machines using spin torque nano-oscillators, Appl. Phys. Lett. 118, 112404 (2021).

[46] A. Vansteenkiste, J. Leliaert, M. Dvornik, M. Helsen, F. Garcia-Sanchez, and B. Van Waeyenberge, The design and verification of MuMax3, AIP Adv. 4, 107133 (2014).

[47] M. Frankowski, J. Chęciński, and M. Czapkiewicz, Spatial spectrum analyzer (SSA): A tool for calculations of spatial distribution of fast Fourier transform spectrum from object oriented micromagnetic framework output data, Comput. Phys. Commun. 189, 207 (2015).

[48] F. Glover, G. Kochenberger, and Y. Du, Quantum bridge analytics i: A tutorial on formulating and using QUBO models, 4OR 17, 335 (2019).

[49] M. Dvornik, A. A. Awad, and J. Åkerman, Origin of Magnetization Auto-Oscillations in Constriction-Based Spin Hall Nano-Oscillators, Phys. Rev. Appl. 9, 014017 (2018).

[50] A. N. Slavin and V. S. Tiberkevich, Theory of mutual phase locking of spin-torque nanosized oscillators, Phys. Rev. B 74, 104401 (2006).

[51] H. Fulara, M. Zahedinejad, R. Khymyn, M. Dvornik, S. Fukami, S. Kanai, H. Ohno, and J. Akerman, Strong voltage-induced tunability of threshold current and frequency in spin Hall nano-oscillators, Bulletin of the American Physical Society (2020).

[52] M. Zahedinejad, H. Fulara, R. Khymyn, A. Houshang, S. Fukami, S. Kanai, H. Ohno, and J. Åkerman, Memristive control of mutual SHNO synchronization for neuromorphic computing (2020).

[53] M. Dvornik, An example of simple Max - Cut problem solved with D - WaveLeapQuantumCloudService (2021).

[54] J. Wu and X. Li, Finite-time and fixed-time synchronization of Kuramoto-oscillator network with multiplex control, IEEE Trans Control Netw. Syst. 6, 863 (2019).

[55] S.-W. Son, H. Jeong, and H. Hong, Relaxation of synchronization on complex networks, Phys. Rev. E 78, 016106 (2008).

[56] V. Flovik, F. Macià, and E. Wahlström, Describing synchronization and topological excitations in arrays of magnetic spin torque oscillators through the Kuramoto model, Sci. Rep. 6, 32528 (2016).

[57] H. Mazraati, S. Chung, A. Houshang, M. Dvornik, L. Piazza, F. Qejvanaj, S. Jiang, T. Q. Le, J. Weissenrieder, and J. Åkerman, Low operational current spin Hall nanooscillators based on NiFe/W bilayers, Appl. Phys. Lett. 109, 242402 (2016).

[58] A. Slavin and V. Tiberkevich, Nonlinear auto-oscillator theory of microwave generation by spin-polarized current, Magn. IEEE Trans. 45, 1875 (2009).

Correction: The author order was set incorrectly and has been fixed. 